\title{
The unresolved challenge of subjects at intermediate cardiovascular risk
}

\author{
Alberto Tosetto \\ Received: 22 April 2011/Accepted: 20 May 2011/Published online: 3 June 2011 \\ (c) SIMI 2011
}

Atherosclerosis is a chronic and progressive disease affecting the arterial vascular bed, particularly large to medium-size arteries at the site of blood flow instability [1]. Stroke and acute myocardial infarction (AMI) are the most severe clinical manifestations of an otherwise silent disease, both resulting in severe disability or death in a significant population proportion. Although the incidence of cerebro or cardiovascular disorders has been steadily decreasing in western countries in the past few decades [2], a success of preventive medicine justifying the need for a stringent control of modifiable risk factors, its burden in the general population is still remarkably high.

The INTERHEART study recently provided evidence from 15,152 AMI cases and 14,820 controls that abnormal plasma lipids, smoking, hypertension, diabetes, obesity, psychosocial factors, consumption of fruits, vegetables, and alcohol, and regular physical activity account for most of the attributable risk for AMI, whatever the gender, age or geographical region [3]. The study clearly shows the multiplicative effect of the presence of several risk factors on cardiovascular risk: while the presence of a single risk factor confers a relative risk of AMI between 2 and 3, presence of three risk factors confers a 15-fold increased risk, and presence of four risk factors a nearly 40-fold increased risk. Indeed, over $90 \%$ of CHD events occur in individuals with at least one risk factor, while the complete absence of major risk factors predicts a very low cardiovascular risk. In an attempt to predict the cardiovascular risk, several regression models have been proposed, the most popular being the Framingham risk score [4]. Based on this prediction model, the National Health and Nutrition

A. Tosetto $(\square)$

Hematology Department, S. Bortolo Hospital, Vicenza, Italy

e-mail: tosetto@hemato.ven.it
Examination Survey (NHANES) III observes that $81.7 \%$ of subjects living in the US have a 10 -year risk for cardiovascular disease $<10 \%$ (low risk), $15.5 \%$ of $10-20 \%$ (intermediate risk), and $2.9 \%$ of $>20 \%$ (high risk) [5]. More appropriately, European guidelines for the prevention of cardiovascular disease consider as at increased risk those subjects having a composite end point of all vascular mortality higher than $5 \%$ in the next 10 years [6].

In clinical practice, however, prediction models often prove to be not entirely satisfactory for several reasons, the most important being that they are very efficient in identifying high-risk subjects, but they tend to misclassify subjects who may have multiple marginal abnormalities. Given the figures of the NHANES III, at least $15.5 \%$ of the subjects may be considered at low risk, especially if young. Therefore, while there is ample amount of evidence that subjects at high cardiovascular risk should be aggressively treated and that subjects without risk factors warrant only for periodic clinical reassessment, we are still in the middle of troubled waters for patients at intermediate cardiovascular risk.

A potential way of improving our prediction model could be the addition of other clinical or biochemical markers in patients within a definite risk category. For instance, the addition of carotid intima-media thickness (cc-IMT) and plaque is found to improve the Framingham risk score in subjects at high vascular risk [7]. Addition of multiple biomarker scores to a traditional risk factor model has also been recently evaluated, and shows some promises [8].

In this issue of the journal, Perego et al. [9] evaluate the presence of target organ damage in a population at intermediate cardiovascular risk. The investigators' aim is essentially to demonstrate the presence of atherosclerotic changes in subjects who usually do not deserve a 
particularly aggressive preventive strategy, and hence they tried to further select the subjects with at least one additional risk factor. Thus, from a population of 11,618 subjects, screened by general practitioners with risk assessment charts, they selected 1,018 subjects at intermediate cardiovascular risk, but having a family history of vascular disease, obesity or heavy smoking. In all these subjects, they assessed cc-IMT and plaque at any level using ultrasound echography, left ventricular mass index (LVMI) by echocardiography, urine albumin/creatinine ratio (UACR), and ankle-brachial index (ABI). In these otherwise healthy subjects, Perego et al. observe a particularly high frequency of such lesions: the overall prevalence of at least one target organ disease was $63 \%$, cc-IMT being increased in $48.2 \%$, UACR abnormal in $14.1 \%$, LVMI high in $12.6 \%$ and ABI pathological in $9.1 \%$.

Given these figures, it may be tempting to propose a two-tier classification of cardiovascular risk: subjects having a low or intermediate risk without additional risk factors should be separated from subjects having an intermediate risk with additional risk factors or high risk, who may benefit from specific interventions. In this regard, the findings of the present report are in keeping with a previous prospective study on an Italian population in which the incidence of cerebrovascular events is higher in patients at intermediate Framingham risk, but with presence of a carotid plaque than in those at high Framingham risk without the presence of carotid plaque as an additional risk factor [7].

Evidence for such a classification is too scarce, however, and the present report has several weaknesses that should also be highlighted. First, the authors used a cross-sectional study design instead of a prospective one, hence not allowing the actual estimation of the risk of vascular events in the subjects at intermediate risk. Second, the authors evaluated some surrogate end-points instead of the relevant ones, i.e. AMI or stroke. Third, the study lacks a clearly defined control group, so that we cannot appreciate the prevalence of target organ damage in subjects at lower risk or at intermediate risk but it is appreciably lower without the additional risk factors.

In conclusion, this study is certainly valuable because it stresses the importance of a complete clinical examination, including the collection of a detailed personal and familial history, in subjects deemed to be at risk of cardiovascular disease. Certainly, an approach solely based on the crude computation of the Framingham score (or other risk scores) is very limited in terms of predictive power, and clinicians feel some urgent need to improve on that. It remains to be established, however, whether the best strategy to improve over and beyond the Framingham score could be based on the additional of non-invasive clinical tests (either additional risk factors, or early markers of disease such as ccIMT) or on arrays of new predictive markers.

Conflict of interest None.

\section{References}

1. Chatzizisis YS, Coskun AU, Jonas M, Edelman ER, Feldman CL, Stone PH (2007) Role of endothelial shear stress in the natural history of coronary atherosclerosis and vascular remodeling: molecular, cellular, and vascular behavior. J Am Coll Cardiol 49:2379-2393

2. Cooper R, Cutler J, Desvigne-Nickens P, Fortmann SP, Friedman L, Havlik R et al (2000) Trends and disparities in coronary heart disease, stroke, and other cardiovascular diseases in the United States: findings of the national conference on cardiovascular disease prevention. Circulation 102:3137-3147

3. Yusuf S, Hawken S, Ounpuu S, Dans T, Avezum A, Lanas F et al (2004) Effect of potentially modifiable risk factors associated with myocardial infarction in 52 countries (the INTERHEART study): case-control study. Lancet 364:937-952

4. D'Agostino RB Sr, Grundy S, Sullivan LM, Wilson P (2001) Validation of the Framingham coronary heart disease prediction scores: results of a multiple ethnic groups investigation. JAMA 286:180-187

5. Ford ES, Giles WH, Mokdad AH (2004) The distribution of 10-Year risk for coronary heart disease among US adults: findings from the National Health and Nutrition Examination Survey III. J Am Coll Cardiol 43:1791-1796

6. Graham I, Atar D, Borch-Johnsen K, Boysen G, Burell G, Cifkova $\mathrm{R}$ et al (2007) European guidelines on cardiovascular disease prevention in clinical practice: executive summary: Fourth Joint Task Force of the European Society of Cardiology and Other Societies on Cardiovascular Disease Prevention in Clinical Practice. Eur Heart J 28:2375-2414

7. Prati P, Tosetto A, Vanuzzo D, Bader G, Casaroli M, Canciani L et al (2008) Carotid intima media thickness and plaques can predict the occurrence of ischemic cerebrovascular events. Stroke 39:2470-2476

8. Blankenberg S, Zeller T, Saarela O, Havulinna AS, Kee F, Tunstall-Pedoe $\mathrm{H}$ et al (2010) Contribution of 30 biomarkers to 10-year cardiovascular risk estimation in 2 population cohorts: the MONICA, risk, genetics, archiving, and monograph (MORGAM) biomarker project. Circulation 121:2388-2397

9. Perego F, Renesto E, Arquati M, Scandiani L, Cogliati C, Torzillo D et al (2011) Target organ damage in a population at intermediate cardiovascular risk, with adjunctive major risk factors: CArdiovascular PREvention Sacco Study (CAPRESS). Intern Emerg Med 